\title{
A meshless method based on Taylor series
}

\author{
Y. Tampango ${ }^{1}$, M. Potier-Ferry ${ }^{1} \&$ Y. Koutsawa ${ }^{2}$ \\ ${ }^{1}$ LEM3, Laboratoire d' Etude des Microstructures et de Mécanique \\ des Matériaux, Université de Lorraine, France \\ ${ }^{2}$ CRP Henri Tudor, Esch-sur-Alzette, Luxembourg
}

\begin{abstract}
We study a meshless method based on Taylor series approximation. This method solves quasi-exactly the Partial Differential Equation (PDE) in the domain. The boundary conditions are applied by using a least square method as proposed by Zhang et al. for stabilizing collocation method. Sometimes, the convergence can only be obtained by splitting the domain in to several subdomains: a new bridging technique is proposed to match several polynomial approximations. Finally, numerical techniques like the Domb Sykes plot permits us to estimate the radius of convergence of the series.

Keywords: meshless method, junction method, convergence analysis, Taylor series expansion.
\end{abstract}

\section{Introduction}

Meshless methods are considered as promising alternatives to overcome the difficulties related to mesh generation. Each class of meshless method can be characterized by the form of the used shape functions, the most common ones being based on moving least square techniques [1], radial functions [2] or fundamental solutions [3]. In this paper we discuss another recent method proposed in Zézé et al. [4], where the shape functions are high degree polynoms that are computed by solving locally the Partial Differential Equation. For instance in the case of the bi-dimensional Laplace equation, this analytical solution leads to a family of polynomial shape functions $\operatorname{Re}(x+i y)^{n}$ and $\operatorname{Im}(x+i y)^{n}$. In this respect the presented method is boundary only, because the PDE is solved quasiexactly inside the domain. This method has several advantages. First the number of shape functions is much smaller than with the p-version of finite elements $[5,6]$. 
Second the partial differential equation is solved quasi-exactly inside the domain so that only the discretization of the boundary is needed. Lastly it can converge very rapidly with the degree ( $\mathrm{p}$-convergence).

The scope of the paper is as follows. First we discuss the way to account for the boundary conditions. It is established that a pure collocation generally diverges and that better results are obtained by applying a least square method as proposed by Zhang et al. [7]. Next a computational technique is sketched that permits to apply Taylor series to PDE's, what permits to compute explicitly the shape functions. Of course, one cannot hope to approximate the solution of any elliptic PDE by a family of polynoms, even if this is possible in theory. That is why we discuss in part 4 bridging techniques to match several Taylor series, each one being valid in a subdomain. In part 5 , the radius of convergence, the location and the nature of the singularities of the sought solution will be analyzed by using classical techniques such as Domb Sykes plot, Hadamard criterion or Darboux criterion [8].

\section{How to apply the boundary conditions}

\subsection{Least square collocation}

In this section, a meshless method based on Taylor series approximation is briefly described. A detailed description of the method may be obtained from Zézé [9]. To illustrate the technique we consider a bi-dimensional Neumann Dirichlet problem:

$$
\left\{\begin{array}{l}
-\Delta u=0 \quad \text { in } \quad \Omega \\
u(\underline{x})=u^{d}(\underline{x}) \quad \text { on } \quad \Gamma_{1} \\
\frac{\partial u}{\partial n}=g \quad \text { on } \quad \Gamma_{2}
\end{array}\right.
$$

where $\partial \Omega=\Gamma_{1} \cup \Gamma_{2}$ and $\Gamma_{1} \cap \Gamma_{2}=\emptyset$.

The main idea of this technique is to introduce high degree polynomial shape functions that are exact or quasi-exact solutions of the PDE. In the case of Laplace equation (1), all the solutions of $-\Delta u=0$ that are polynoms of degree lower or equal to $N$ can be written as:

$$
\begin{gathered}
u(x, y)=\sum_{k=1}^{2 N+1} q_{k} P_{k}(x, y)=\langle P(x, y)\rangle\{q\} . \\
\langle P(x, y)\rangle=\left\langle 1, x, y, x^{2}-y^{2}, x y, \cdots, \operatorname{Re}(x+i y)^{n}, \operatorname{Im}(x+i y)^{n}\right\rangle .
\end{gathered}
$$

So the general solution of the problem is completely obtained by determining these $2 N+1$ variables from the boundary conditions. A least square method combined with collocation method will be used. This technique has been suggested by Zhang et al. [7]. One chooses a set of nodes $\underline{x}_{j}$ on the boundary of the domain and one minimizes the error between the approximate value and the given value of 
$u$ in these points. It comes to minimize the function:

$$
J(v)=\frac{1}{2} \sum_{\underline{x}_{j} \in \Gamma_{1}}\left|u^{h}\left(\underline{x}_{j}\right)-u^{d}\left(\underline{x}_{j}\right)\right|^{2}+\frac{1}{2} \sum_{\underline{x}_{j} \in \Gamma_{2}}\left|\frac{\partial u^{h}}{\partial n}\left(\underline{x}_{j}\right)-g\left(\underline{x}_{j}\right)\right|^{2} .
$$

This minimization leads to a linear system $K\{v\}=F$ where $K$ is an invertible matrix. Solving this system gives the vector $\{v\}$ and therefore the approximate solution of the problem (1).

\subsection{Convergence}

This section presents the numerical solutions of some examples studied to validate the meshless method presented above. The validation will be made on Laplace equation. The Laplace equation has been considered in a circular domain with an exact solution which presents a singularity at a point out of the domain. The effect of the singularity on the solution accuracy was investigated. A comparison of the proposed method with the finite element method will be presented.

We consider the Dirichlet problem (1) in a circular domain:

$$
\left\{\begin{array}{l}
-\Delta u=0 \quad \text { in } \quad \Omega=\left\{(x, y) / x^{2}+y^{2} \leq 1\right\} \\
u(\underline{x})=\frac{x-x_{0}}{\left(x-x_{0}\right)^{2}+\left(y-y_{0}\right)^{2}} \quad \text { on } \quad \partial \Omega .
\end{array}\right.
$$

Here we are interested by the influence of the singular point $X_{0}=\left[x_{0}, y_{0}\right]$ on the rate of convergence. For that we examine the maximum of the relative error in the domain. The relative error is defined by:

$$
E=\frac{\left|u(\underline{x})-u^{h}(\underline{x})\right|}{|u(\underline{x})|}=\frac{\left|u(\underline{x})-\sum_{i=0}^{N} u_{i, N-i} x^{i} y^{N-i}\right|}{|u(\underline{x})|} .
$$

Let us examine $\mathrm{E}$ for two different singular points.

From Fig. 1, the following comments can be made.

- The algorithm converges. The error is about $10^{-10}$ for the singular point at $X_{0}=[2,0.3]$ and for order $p=30$.

- The technique converges exponentially with the degree ( $\mathrm{p}$-convergence).

- The quality of the approximation depends on the considered problem, especially on the position of the singular point $X_{0}$. The rate of convergence is better if the singularity is away from the domain.

The same method has been also applied to various boundary conditions (Neumann, Robin) and to various PDE (Helmholtz equation, linear elasticity), and various domains, similar conclusions have generally been obtained.

The present results have been compared with the finite element method. Roughly, our boundary meshless requires much less degrees of freedom. For instance, the problem (5) with $X_{0}=[1.2,0.3]$ can be solved with a relative error of $10^{-4}$ with only 91 dof's, while quadrangular finite elements of degrees 2 require 4880 dof's and triangular linear FE need 42000 dof's. 


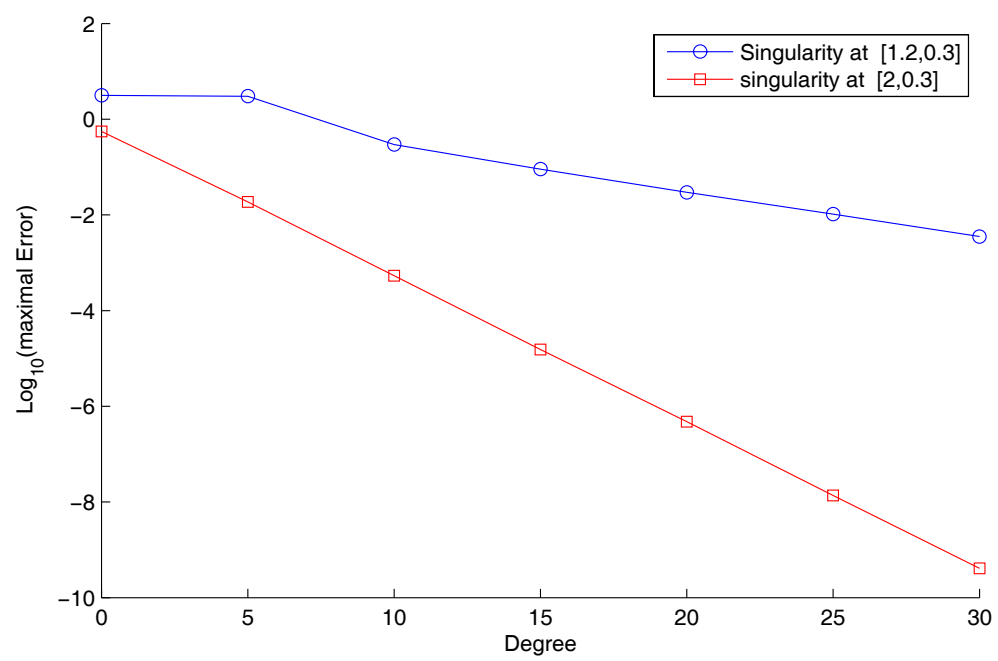

Figure 1: P-convergence for two Laplace problems (5) that differ by the position of the singularity $X_{0}=\left[x_{0}, y_{0}\right]$.

As established in [4, 9], the pure collocation approach does not converge in many cases: the least square collocation method is necessary in the case of high degree polynomial shape functions.

Last the present method does not converge in any case, especially for annular domains with an exact solution that has a singularity inner the small circle. That is why a splitting in subdomains is sketched in part 4 .

\section{How to compute the shape functions}

The basic idea is to consider the PDE as an ordinary differential equation with respect to one variable, say $y$, and to solve the Cauchy problem with initial data $u(x, 0)$ and $\partial u / \partial y(x, 0)$. These initial data will be approximated by polynoms with respective degrees $N$ and $N-1$ and $u(x, y)$ is sought in the form of a polynom of degree $N$.

To illustrate the technique, let us considered the homogeneous Helmholtz equation with constant coefficients:

$$
-\Delta u+c u=0
$$

The approximate solution of the PDE (7) is sought in the form of a polynom of degree $N$ :

$$
u^{h}(x, y)=\sum_{k=0}^{N} \sum_{i=0}^{k} u_{i, k-i} x^{i} y^{k-i}=\sum_{k=0}^{N}\left\langle X^{k}\right\rangle\left\{u^{k}\right\}
$$




$$
\left\langle X^{k}\right\rangle=\left\langle x^{k}, x^{k-1} y, \cdots, y^{k}\right\rangle, \quad{ }^{t}\left\{u^{k}\right\}=\left\langle u_{k, 0}, u_{k-1,1}, \cdots, u_{0, k}\right\rangle .
$$

For each degree $k$, the unknown is the vector $\left\{u^{k}\right\} \in \mathbb{R}^{k+1}$. For the complete polynom (8), there are $(N+1)(N+2) / 2$ coefficients to be found.

By vanishing the polynom (7), one gets a linear system of equations:

$$
-\left[\Delta^{k}\right]\left\{u^{k+2}\right\}+c\left\{u^{k}\right\}=\{0\} \quad \forall k, 0 \leq k \leq N-2
$$

For each $k$, there are $k+1$ equations in (9) for $k+3$ unknowns $\left(\left\{u^{k+2}\right\} \in \mathbb{R}^{k+3}\right)$. Each vector $\left\{u^{k+2}\right\}$ can then be written as a function of its two first components:

$$
\left\{v^{k}\right\}=\left\{\begin{array}{l}
u_{k, 0} \\
u_{k-1,1}
\end{array}\right\} \in \mathbb{R}^{2} .
$$

Hence the account of the PDE (see [4]) reduces the general polynom (8) to a family of $2 N+1$ polynoms $\left\langle P_{k}>\right.$ instead of $(N+1)(N+2) / 2$ :

$$
u^{h}(x, y)=\sum_{k=0}^{N}\left\langle P_{k}>\left\{v^{k}\right\}=\langle P(x, y)\rangle\{v\} .\right.
$$

The procedure can be extended in more complex cases, for instance if $c$ is a function of $(x, y)$, see [4]. One gets equations similar to (9) by requiring that all the derivatives of $-\Delta u+c u$ at a point $\left(x_{c}, y_{c}\right)$ vanish up to order $N-2$.

In order words, the vectorial space (11) contains all the polynoms that are approximated solutions of the PDE, the approximation made understood in the sense of Taylor series at a given expansion point.

An easy implementation of this algorithm can be established with the help of Automatic Differentiation method [10].

\section{Towards a piecewise discretisation method}

The boundary meshless method described in Part 2 does not converge in any case. For instance, we have studied the Poisson problem in an annular domain:

$$
\left\{\begin{array}{l}
-\Delta u=\frac{1}{\left(x^{2}+y^{2}\right)^{2}} \quad \text { in } \quad \Omega=\left\{(x, y) / r_{1}^{2} \leq x^{2}+y^{2} \leq r_{2}^{2}\right\} \\
u(\underline{x})=u^{d}(\underline{x}) \quad \text { on } \quad \Gamma_{1}
\end{array}\right.
$$

For this problem and for any problem, whose solution has a singularity inside the small circle, the method of Part 2 diverges.

To overcome the difficulty, we propose to seek the solution in a piecewise manner, as in the finite element method.Thus the domain is split in several subdomains and a polynomial solution in the form (8) is introduced in each subdomain. How to match these high degree polynoms? Of course it is not possible to achieve a perfect continuity along the interface. A first idea, presented in [9], is to introduce new terms in the minimization problem (4) to account for the 


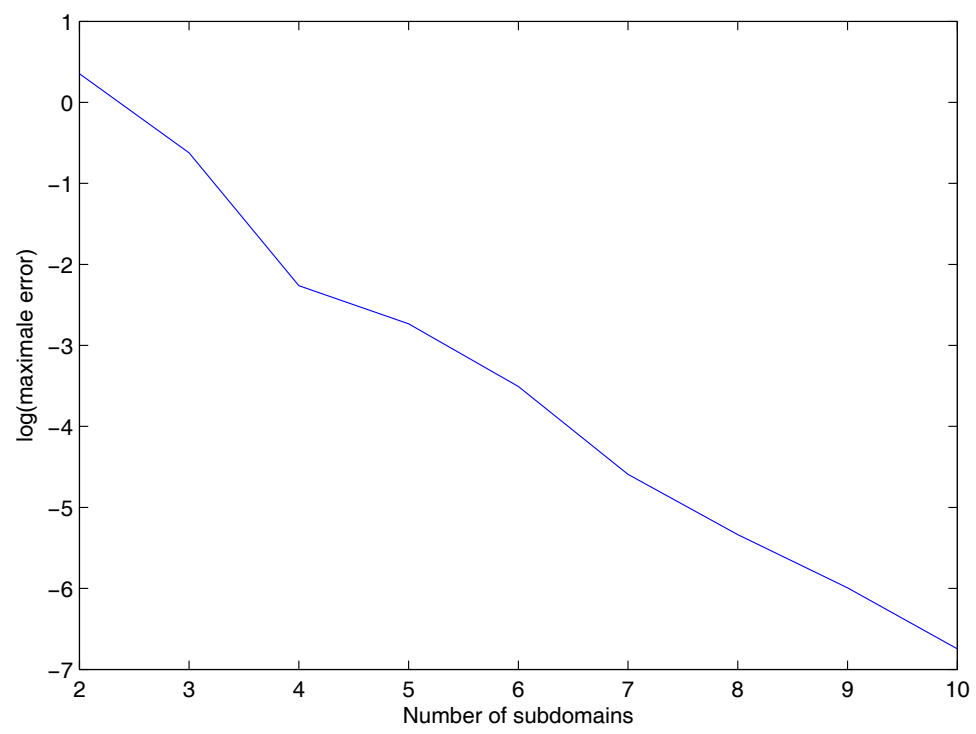

Figure 2: Influence of the number of subdomains (h-convergence).

continuity of $u$ and its normal derivative along the interface. The latter method is able to provide correct approximation (say error about 1\%), but it is not reliable and does not ensure an exponential convergence with the degree.

Here we present another idea, where the continuity of $u$ and $\partial u / \partial n$ at some collocation points $\underline{x}_{j}$ along the interface is introduced as constraints in the minimization problem (4):

$$
h_{1}(\underline{x})=u^{1}(\underline{x})-u^{2}(\underline{x}) \quad, \quad h_{2}(\underline{x})=\frac{\partial u^{1}}{\partial n}(\underline{x})-\frac{\partial u^{2}}{\partial n}(\underline{x}) .
$$

In the case of two subdomains, the discrete problem to be solved follows from the stationarity of the function:

$$
L\left(v_{1}, v_{2}, \lambda_{1}, \lambda_{2}\right)=J_{1}\left(v_{1}\right)+J_{2}\left(v_{2}\right)+\sum_{i}\left(\lambda_{1}^{i} h_{1}\left(\underline{x}_{i}\right)+\lambda_{2}^{i} h_{2}\left(\underline{x}_{i}\right)\right) .
$$

As an example, let us study the Poisson problem in a crown of radius $r_{1}=0.8$, $r_{2}=1$. The convergence with the number of subdomains (h-convergence) is presented in figure 2 in the case of a degree $N=10$ : one sees that a monotone convergence and a high accuracy (error of about $10^{-6}$ ) can be obtained in this way.

An exponential convergence is obtained for a number of subdomains larger than 4 . For instance, with four subdomains and a degree 20, the relative error is of $10^{-4}$. 
It is also possible to introduce continuity constraints in a mean sense, in a way similar to the Arlequin method [11]. This leads to very good results that will be presented in forthcoming papers.

\section{Evaluation of the convergence radius and convergence acceleration}

As seen in figure 1, the speed of convergence depends on the considered problem. Hence there is a need to analyze the convergence of Taylor series and to find the location and the nature of singularities of the function computed by the high order meshless method.

\subsection{Radius of convergence}

Generally the radius of convergence of a power series is determined by the behavior of its coefficients at infinity. There exist several techniques to estimate the radius of convergence from a finite number of coefficients $[12,13]$. The radius of convergence is supposed to be the nearest singularity. We applied some of these techniques to our algorithm and we compare the results to the Hadamard criterion which postulates that "the radius of convergence of a series $\sum a_{n} x^{n}$ is $R=1 / L$ with $L=\lim \sup \left\|a_{n}\right\|^{1 / n}$ ".

\subsection{Domb Sykes plot}

Domb and Sykes in [12] shows that the inverse ratio $c_{n} / c_{n-1}$ is often a linear function of $1 / n$ for $n$ sufficiently large. Indeed for a singular function of type (singularity at $x_{0}$ ):

$$
f(x) \cong \text { const } *\left\{\begin{array}{l}
\left(x_{0} \pm x\right)^{\nu}, \quad \nu \neq 0,1,2, \cdots \\
\left(x_{0} \pm x\right)^{\nu} \log \left(x_{0} \pm x\right), \quad \nu=0,1,2, \cdots
\end{array}\right.
$$

the coefficients of its power series satisfy:

$$
D_{s}(i) \cong \frac{c_{i}}{c_{i-1}}= \pm \frac{1}{x_{0}}\left(1-\frac{1+\nu}{i}\right) .
$$

A similar method, attributed to Darboux, can also be applied [13].

\subsection{Padé approximant}

The idea of this technique is to replace a power series truncated at degree $M+N$ by a rational function $[M / N]$ with a numerator of degree $M$ and a denominator of degree $N$ [14]. In the literature, Padé approximants are used for convergence acceleration and for detection of singularities [15]. The singularities are detected by the poles of the fraction. 
Let $f$ be an analytic function defined by a formal power series:

$$
f(z)=\sum_{i=0}^{\infty} c_{i} z^{i}
$$

A $[L / M]$ Padé approximant of the function $\mathrm{f}$ is a rational fraction such that the Taylor expansion coincides with the one of $f$ up to order $L+M$.

The advantages and drawbacks of Padé approximants are well known. As compared with the previous methods, it can detect several singularities, often accurately, but not an order of singularity that differs from -1 . Nevertheless this procedure is not quite reliable. Some insignificant poles can be observed within the computations of Padé approximants, that are called "defects" in the Padé literature [14].

\subsection{Numerical results}

In this section, the radius of convergence and the nearest singularity are evaluated by using the classical criteria presented in the previous sections. Let us recall that we do not analyse a given analytical function, but a numerical function that is obtained by combining Taylor series and boundary collocation, as presented in section 2. In other words, one looks at a possible influence of the identification by collocation on the radius of convergence of the solution of the PDE. Indeed the strong connection between singularity and exponential convergence of the method has been established in section 2 and this evaluation of the nearest singularity should permit to predict the convergence of the boundary collocation method.

Three techniques have been presented to analyse the Taylor series: the Hadamard criterion that leads to an estimate of the radius of convergence, the Domb Sykes plot that gives the nearest real singularity and its order and the Padé approximant method that permits to find some singularities and get convergence improvements. All these methods analyse univariate functions, while the solution of the PDE is a function of two variables $u(x, y)$. The four convergence criteria will be applied on some lines of the plane, for instance lines parallel to $o x$ by considering the function $x \rightarrow u(x, y)$ or radial lines in the $\theta$ direction by considering $r \rightarrow u(r \cos \theta, r \sin \theta)$.

We have applied the Domb Sykes plot in several directions and we observed that the ratio $D_{s}(N)$ converges only in the direction of the singularity $\theta=$ $\arctan \left(y_{0} / x_{0}\right)$.

Hence it seems that an efficient algorithm to detect the nearest singularity in the plane would be defined by analysing the ratio $D_{s}(N)$ in many radial lines. The direction of the singularity is the one such that the ratio $D_{s}(N)$ converges for large $N$ :

$$
\left\|D_{s}(N)-D_{s}(N-1)\right\| \leq \epsilon
$$

In table 1 , one presents the position of the singularity estimated by Hadamard criterion and Domb Sykes plot. This table is built in the direction of the singularity. 
It is found that these two techniques give a good estimation of the position of singularity as well as the order of singularity.

Table 1: Position of singularity.

Table 2: Padé approximant poles.

\begin{tabular}{ccc}
\hline $\begin{array}{c}\text { Degree } \\
(\mathrm{N})\end{array}$ & $\begin{array}{c}\text { Domb } \\
\text { Sykes }\end{array}$ & $\begin{array}{c}\text { Hadamard } \\
\text { criterion }\end{array}$ \\
\hline 15 & 1.2374 & 1.2395 \\
20 & 1.2368 & 1.2388 \\
30 & 1.2369 & 1.2381 \\
40 & 1.2369 & 1.2378 \\
\hline
\end{tabular}

\begin{tabular}{ccc}
\hline Padé & $\theta=0$ & $\theta=\arctan \left(y_{0} / x_{0}\right)$ \\
\hline$[11 / 1]$ & 1.2109 & 1.2346 \\
{$[10 / 2]$} & $1.2 \pm 0.29 \mathrm{i}$ & $1.2369 ; 0.808$ \\
{$[6 / 6]$} & $1.2 \pm 0.3 \mathrm{i}$ & $1.2369 ; 0.808$ \\
& $0.78 \pm 0.19 \mathrm{i}$ & $-0.04 \pm 1.15 \mathrm{i}$ \\
& $-0.82 \pm 0.85 \mathrm{i}$ & $-0.9 \pm 0.62 \mathrm{i}$ \\
\hline
\end{tabular}

In table 2 we present the poles of the Padé approximant for the Laplace problem in several directions.

In contrast to the results from Domb Sykes method, the singular point appears whatever the chosen direction. Despite all, this pole is difficult to be extracted in cases where the structure of the analytical solution is unknown. This is due to the presence of other poles, which are known as defects by specialists of Padé approximants (see for instance the pole 0.808 in the last column of table 2). Hence this technique can not be used alone to define a reliable criterion for detecting singularity.

But in the literature, Padé approximant method is one of the most common methods for improving the convergence of a series [15]. A comparison between the accuracy of the polynomial approximation and of the Pade approximants is presented in table 3. Clearly the Padé approximants improves significantly the convergence of the method (error of order $10^{-3}$ instead of $10^{-1}$ with series).

Table 3: Logarithm of the error with various Padé approximants: Laplace problem with singularity at $[1.2,0.3]$.

\begin{tabular}{lccccc}
\hline & $\begin{array}{c}\text { Series } \\
(\text { degree }=12)\end{array}$ & $\begin{array}{c}\text { Pade } \\
{[11 / 1]}\end{array}$ & $\begin{array}{c}\text { Pade } \\
{[10 / 2]}\end{array}$ & $\begin{array}{c}\text { Pade } \\
{[9 / 3]}\end{array}$ & $\begin{array}{c}\text { Pade } \\
{[6 / 6]}\end{array}$ \\
\hline$\theta=0$ & -1.048 & -0.676 & -2.417 & -2.415 & -4.188 \\
$\theta=\pi / 2$ & -1.561 & -1.2025 & -4.313 & -4.333 & -4.568 \\
$\theta=-2 \pi / 3$ & -1.547 & -0.459 & -3.484 & -2.798 & -4.408 \\
$\theta=\arctan \left(y_{0} / x_{0}\right)$ & -0.591 & -2.255 & -2.776 & -2.776 & -2.776 \\
\hline
\end{tabular}




\section{Concluding remarks}

Some numerical tools have been presented to evaluate the convergence properties of Taylor series computed by a high order boundary meshless method. The key point of this meshless method is the introduction of high degree polynomial shape functions that are accurate solutions of the studied PDE. The principle is to require that the Taylor coefficients of the equation at a given point vanish. This is a true boundary meshless method, the discrete equations being obtained by a least squares collocation technique and the collocation points located only on the boundary. A comparison with the classical finite element method has shown that the present method requires much less degrees of freedom.

It has been clearly established that the method converges exponentially with the degree and that the speed of convergence is strongly related to the radius of convergence and to the location of the nearest singularity. For simple problems, the method may converge with a single polynomial approximation. Nevertheless, to build a generic numerical technique, one has to split the domain in several subdomains. This is a difficult challenge to match efficiently high degree approximations: a new collocation technique involving Lagrange multipliers has been proposed that preserves the ability to converge. Several classical tools to evaluate the convergence of the series have been tested for academic boundary value problems.

\section{Acknowledgements}

This research was financially supported by FNR Luxembourg through AFR Grant PHD-09-017. The authors also would like to gratefully thank CRP Henri Tudor Luxembourg for their technical support.

\section{References}

[1] Nayroles, B., Touzot, G. and Villon, P., Ageneralizing the finite element method: Diffuse approximation and diffuse elements. Computational Mechanics, 10, pp. 307-318, 1992.

[2] Kansa, E., A scattered data approximation shame with applications to computational fluid dynamics. I. surface approximations and partial derivative estimate. Computer and Mathematics with Applications, 19, pp. 127-145, 1990.

[3] Golberg, M., The method of fundamental solutions for poisson's equation. Engineering Analysis with Boundary Elements, 16, pp. 205-213, 1995.

[4] Zézé, D., Potier-Ferry, M. and Damil, N., A boundary meshless method with shape functions computed from the pde. Engineering Analysis with Boundary Elements, 34, pp. 747-754, 2010.

[5] Babuska, I., Szabo, B. and Katz, L., The p-version of the finite element method. SIAM Journal on Numerical Analysis, 18, pp. 515-545, 1981. 
[6] Campion, S. and Jarvis, J., An investigation of the implementation of the pversion finite element method. Finite Element in Analysis and Design, 23, pp. 1-21, 1996.

[7] Zhang, X., Liu, X., Song, K. and Lu, M., Least squares collocation meshless method. International Journal for Numerical Methods in Engineering, 51, pp. 1089-1100, 2001.

[8] Tampango, Y., Potier-Ferry, M., Koutsawa, Y. and Belouettar, S., Convergence analysis and detection of singularities within a boundary meshless method based on Taylor series. Engineering Analysis with Boundary Elements, In press, 2012.

[9] Zézé, D., Calcul de fonctions de forme de haut degré par une technique de perturbation. Ph.D. thesis, Université Paul Verlaine Metz, 2009.

[10] Griewank, A., Evaluating derivatives: principle and techniques of algorithmic differentiation. SIAM: Philadelphia, 2000.

[11] Bendhia, H. and Rateau, G., The Arlequin method as a flexible engineering tool. International Journal for Numerical Methods in Engineering, 62, pp. 1442-1462, 2005.

[12] Domb, C. and Sykes, M., Use of series expansions for the ising model susceptibility and excluded volume problem. Journal of Mathematical Physics, 2, pp. 63-67, 1961.

[13] Hunter, C. and Guerrieri, B., Deducing the properties of singularities of functions from their Taylor series coefficients. SIAM Journal on Applied Mathematics, 39, pp. 248-263, 1980.

[14] Baker, P., G.A.and Graves-Morris, Padé Approximants. Cambridge University Press: New York, 1996.

[15] Van Dyke, M., Analysis and improvement of perturbation series. Quarterly Journal of Mechanics and Applied Mathematics, 27, pp. 423-450, 1974. 